\title{
Towards a heuristic understanding of the storage effect
}

\author{
Evan Johnson ${ }^{1}$ and Alan Hastings ${ }^{2}$ \\ ${ }^{1}$ University of California Davis \\ ${ }^{2}$ University of California
}

February 14, 2022

\begin{abstract}
The storage effect is a general explanation for coexistence in a variable environment. The generality of the storage effect is both a strength - it can be quantified in many systems - and a challenge - there is not a clear relationship between the abstract conditions for storage effect and species' life-history traits (e.g., dormancy, stage-structure, non-overlapping generations), thus precluding a simple ecological interpretation of the storage effect. Our goal here is to provide a clearer understanding of the conditions for the storage effect as a step towards a better general explanation for coexistence in a variable environment. Our approach focuses on dividing one of the key conditions for the storage effect, covariance between environment and competition, into two pieces, namely that there must be a causal relationship between environment and competition, and that the effects of the environment do not change too quickly. This finer-grained definition can explain a number of previous results, including 1) that the storage effect promotes annual plant coexistence when the germination rate fluctuates, but not when the seed yield fluctuates, 2) that the storage effect is more likely to be induced by resource competition than apparent competition, and 3) that the spatial storage effect is more probable than the temporal storage effect. Additionally, our expanded definition suggests two novel mechanisms by which the temporal storage effect can arise: transgenerational plasticity, and causal chains of environmental variables. These mechanisms produce coexistence via the storage effect without any need for stage structure or a temporally autocorrelated environment.
\end{abstract}

\section{Hosted file}

letters.pdf available at https://authorea.com/users/460380/articles/556407-towards-aheuristic-understanding-of-the-storage-effect 